Journal of Engineering and Applied Sciences 5 (3): 211-220, 2010

ISSN: 1816-949X

(C) Medwell Journals, 2010

\title{
Numerical Simulation of a Lifted Methane Jet Flame in a Vitiated Coflow: Lagrangian Approach with Detail Chemistry
}

\author{
${ }^{1,2}$ Ruben Mouangue, ${ }^{1}$ Marcel Obounou, ${ }^{1}$ Donatien Njomo and ${ }^{1}$ Cyrille Gnemtedem \\ ${ }^{1}$ Department of Physics, Faculty of Sciences, University of Yaoundé 1, P.O. Box 812, Cameroon \\ ${ }^{2}$ Department of Energetic Engineering, University of Ngaoundéré, IUT, P.O. Box 455, Cameroon
}

\begin{abstract}
Numerical calculation of turbulent lifted flame is studies. In this study, mass flow rate, temperature and exact chemical composition of hot products mixed with air sent toward the turbulent flame base are fully determined. The effects of both non-infinitely fast chemistry and partially premixed combustion are taken into account by using the MIL model (Modèle Intermittent Lagrangien) originally introduced by Borghi and Gonzalez with complex chemistry. Here, the model based on a presumed joint PDF (Probability Density Function) shape for both a mixture fraction and a mixing time for production of a reaction. A fully detailed chemistry are construct about the tabulation of ignition delays and introduce in calculation of the mean chemical rate; this allows us to express the higher probability that fluid particles have to burn for large range around stoichiometric composition. Numerical simulation of the turbulent diluted jet flame of methane studied by R. Cabra and his co-workers at Berclays University is satisfactory; the lift of height prediction are successfully compared with experiments and was around 30-40 D.
\end{abstract}

Key words: Numerical simulation, fine rate chemistry, lifted flame, detailed chemistry, partially premixed turbulent combustion, Cameroon

\section{INTRODUCTION}

In many practical combustion systems the fuel jets emerge into a hot environment of oxidants and combustion products. These hot products carry thermal energy that may help flame stabilization; they are also useful to dilute the reaction zones which homogenizes the mixture and avoids the high temperature levels responsible for $\mathrm{NO}_{\mathrm{x}}$ emissions. Prediction of turbulent jet flames with complex recirculating flows and autoignition mechanisms pose a great challenge to the current combustion modeling. The vitiated coflow flame is a kind of turbulent reacting flow within a hot environment and low oxygen concentration.

Some studies related to the vitiated coflow from the experimental point of view, research of Cabra et al. (2002, 2005) with like fuel a mixture of $\mathrm{H}_{2} / \mathrm{N}_{2}$ then $\mathrm{CH}_{4} /$ air, respectively constitute the reference now-a-days; moreover, Gordon et al. (2008) brought temperature measurements of $\mathrm{OH}$ and of $\mathrm{CH}_{2} \mathrm{O}$ in methane combustion. Calculations were also carried out on this type of configuration by the model of PDF calculated, Gordon et al. $(2007,2008)$ simulated the combustion of two fuels mentioned earlier and brought information on transport by convection-diffusion of the molecules;
Cao et al. (2005) applied its calculation to the only case of $\mathrm{H}_{2} / \mathrm{N}_{2}$, similar to Masri et al. (2003) detailed chemistry was taken into account by use of ISAT method (in situ Adaptive Tabulation). A calculation with $\mathrm{CMC}$ model (Conditional Moment Closure) was carried out by Patwardhan et al. (2009) where an analysis of sensitivity of the zone of pre-flame was question; another of Michel et al. (2009) by use of the flammelettes model ADF-PCM where a accent was laid on the study of the diffusion zone thanks to the correction made on the probability distribution of the scalar dissipation rate at stoichiometry $\mathrm{P} \quad\left(\chi_{s t}\right)$ like a log-normal function. Domingo et al. (2008) carried out a calculation using LES (Large Eddy Simulation) adding an analysis on the turbulent flame and a new closing of the scalar dissipation rate of the reactive species, then Wang et al. (2007) a calculation using the DNS (Direct Numerical Simulation) where an examination of the dynamics of the swirls, structure of the micro-scales and the distribution of the turbulence intensity in the flame are made.

The Lagrangian models in fact the MIL (Modèle Intermittent Lagrangien) model was introduced into the modeling of turbulent combustion for the comprehension of the turbulence-chemistry interaction met in nonpremixed flames (Borghi and Gonzalez, 1986; Gonzalez

Corresponding Author: Marcel Obounou, Department of Physics, Faculty of Sciences, University of Yaoundé 1, P.O. Box 812, Cameroon 
and Borghi, 1991). A well-known projection was observed when Obounou et al. (1994) there introduced detailed chemistry, thus predicting zones of partial extinction then of re-ignition. Gonzalez et al. (1997) applied the ML model within the framework of stabilization to the exit of a burner. This model consists of a formulation of presumed $\mathrm{PDF}$, associates ignition times and makes it possible to follow the history of a particle.

The objective of this study is to apply the MLL model, version developed at the Department of Physics of the University of Yaounde 1 with taking into account of a complex chemistry, then to couple it in a computer code for the simulation of a lifted flame. The study relates to measurements of Cabra et al. (2005) which provides several interesting data with initial and boundary conditions quite exact.

\section{MATERIALS AND METHODS}

Experimental configuration: The exact chemical composition and mass flow rate of recirculating burnt gases are often difficult to calibrate and measure accurately in real combustion chambers. To gain greater understanding of flame stabilization in environments where burnt gases dominate, Cabra et al. (2005) designed a laboratory vitiated burner shown in Fig. 1, in which the injection conditions of reactants and hot products are fully determined. It consists of an $\mathrm{CH}_{4} /$ air fuel jet issuing into a hot coflow which is the combustion product of a lean premixed $\mathrm{H}_{2}$ /air flame. The diameter of the central jet is $\mathrm{d}=4.57 \mathrm{~mm}$; the coflow diameter about $210 \mathrm{~mm}$ is much larger than the central jet which can isolate the central jet from ambient air for a sufficiently long distance.

The corresponding inlet conditions in fuel stream and coflow are shown in Table 1 in terms of mole fractions, temperature, velocity and Reynolds numbers. The RamanRayleigh technique was used for measuring instantaneous mass fractions and temperature; all the mean and instantaneous values have been downloaded from Cabra et al. (2002).

Description of the ML model: The combustion being the seat of two phenomena which are chemistry and turbulence, the interaction of the latter requires an adequate modeling. The $\mathrm{MLL}$ model takes part by its formulation with the comprehension of this type of combustion integrating this competition at the base, this model was introduced for the modeling of the average reaction rate for non-premixed flames (Borghi and Gonzalez, 1986; Gonzalez and Borghi, 1991; Obounou et al., 1994; Gonzalez et al., 1997).

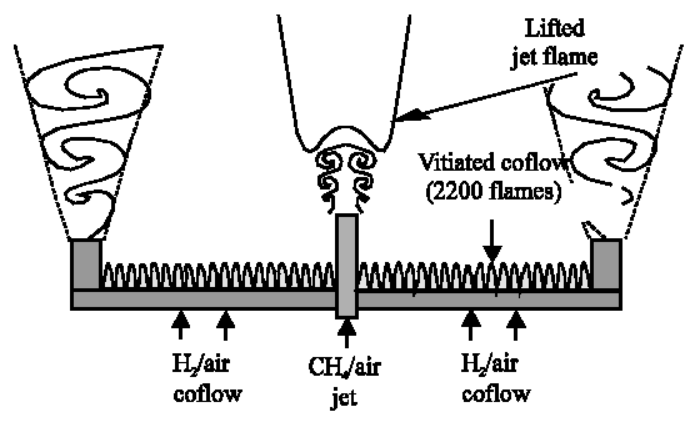

Fig. 1: Experimental arrangement of the Cabra et al. (2005) experiment

Table 1: Boundary conditions of the Cabra et al. (2005) experiment

\begin{tabular}{|c|c|c|c|c|c|c|c|c|}
\hline$\underline{\text { Items }}$ & $\mathrm{R}_{\varepsilon}$ & $\begin{array}{l}\mathrm{D} \\
(\mathrm{mm})\end{array}$ & $\begin{array}{l}\mathrm{U} \\
\left(\mathrm{m} \mathrm{sec}^{-1}\right)\end{array}$ & $\mathrm{T}(\mathrm{K})$ & $\mathrm{X}_{\mathrm{O}_{\mathrm{z}}}$ & $\mathrm{X}_{\mathrm{N}_{z}}$ & $\mathrm{X}_{\mathrm{H}_{2} \mathrm{O}}$ & $\mathrm{X}_{\mathrm{CH}_{+}}$ \\
\hline Fuel jet & 28,000 & 4.57 & 100 & 320 & 0.15 & 0.52 & 0.0029 & 0.3300 \\
\hline Coflow & 23,300 & 210 & 5.4 & 1350 & 0.12 & 0.73 & 0.1500 & 0.0003 \\
\hline
\end{tabular}

Lagrangian paths in the composition space: Therefore of the aerothermochemistry, the equation of the mass fraction of the species is:

$$
\frac{\partial \rho Y_{i}}{\partial t}+\frac{\partial \rho u_{i} Y_{i}}{\partial x_{i}}=\underbrace{\frac{\partial}{\partial x_{k}}\left(\rho D \frac{\partial Y_{i}}{\partial x_{i}}-\rho\left(u_{i} Y_{i}\right)\right)}_{\text {II }}+\underbrace{\rho \omega_{Y}}_{\text {III }}
$$

This equation is in the non-stationary form; (I) convection; (II) diffusion; (III) source; (IV) the term of diffusion being subdivided in molecular diffusion and turbulent diffusion. While returning the term of turbulent diffusion in the member of left which will be paired at the term of convection, all the term of LHS (Left Hand Side) results in a Lagrangian derivative since moreover, the idea is to follow the history of a particle. The laminar term of diffusion (molecular) is modelled by IEM (Interaction par Echange avec la Moyenne) of Villermaux and Devillon (1972); the researchers associate it an equation of the mixing fraction $\phi$ :

$$
\frac{\mathrm{dY}}{\mathrm{dt}}=\frac{\tilde{\mathrm{Y}}-\mathrm{Y}}{\tau}+\omega_{\mathrm{Y}} \text { and } \frac{\mathrm{d} \phi}{\mathrm{dt}}=\frac{\tilde{\phi}-\phi}{\tau}
$$

For a time of exchange shown $\tau$, the resolution of the Eq. 2 lead to the trajectories of the MIL model in the space of the phases; the assumption of sudden chemistry allows the particles burnt after a certain time, thus leaving branch of mixture by a jump to arrive on the equilibrium branch as shown in Fig. 2. On lines IEM, the reaction rate can be neglected, the previous system shows: 


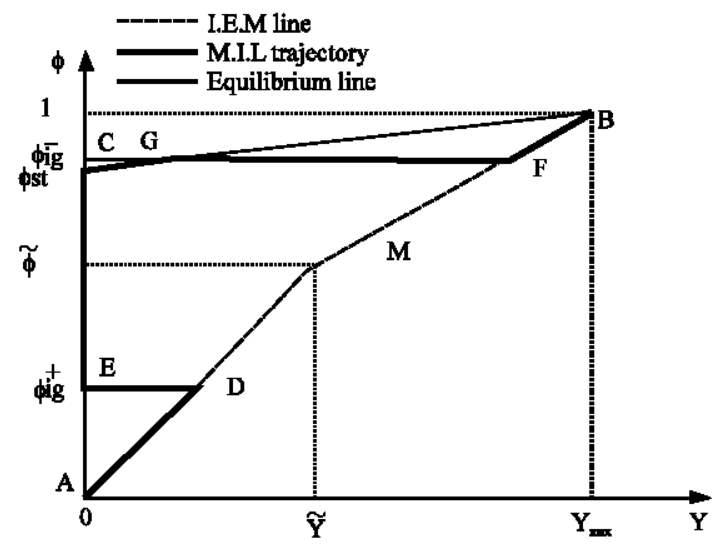

Fig. 2: Conventional representation of the MLL model (thick lines) in the phase plane $(\varphi, Y)$. Equilibrium (plain line), IEM mixing lines (dashed lines) are also depicted

$$
\frac{d Y}{d \phi}=\frac{\tilde{Y}-Y}{\tilde{\phi}-\phi}
$$

Integration gives the branches $\mathrm{AM}$ and $\mathrm{BM}$ of Fig. 2. The particles need a finite time before ignition; they follow trajectories IEM ( $\mathrm{AD}$ and $\mathrm{BF}$ ); then after the ignition occurs, they reach the equilibrium branches instantaneously (DE and FG). Through the simple form of the MLL model, the trajectories in the composition space are regarded as straight segments; $\phi_{i g}^{+}$and $\phi_{\mathrm{ig}}^{-}$represent the values of corresponding to the ignition of the particles coming from pure oxidant and pure fuel not $\mathrm{A}$ and $\mathrm{B}$, respectively from Fig. 2 .

On the equilibrium branch, there are $\mathrm{Y}=0$ from pure oxidant then from pure fuel following expression $\mathrm{Y}=$ $\mathrm{Y}_{\max } / 1-\phi_{\mathrm{st}}\left(\phi-\phi_{\mathrm{st}}\right)$ what corresponds respectively to lines $\mathrm{BC}$ and $\mathrm{AC}$. On the equilibrium branch:

$$
\mathrm{Y}(\phi)= \begin{cases}\frac{\tilde{Y}}{\tilde{\phi}} \phi & \text { If } \phi<\tilde{\phi} \\ \frac{\phi-1}{\tilde{\phi}-1} \tilde{Y}+\frac{\tilde{\phi}-\phi}{\phi-1} \mathrm{Y}_{\operatorname{Max}}, & \text { elsewhere }\end{cases}
$$

Mean production rates: The instantaneous reaction rate is reduced to a function of two variables:

$$
\omega \mathrm{Y}_{\mathrm{i}}=\omega \mathrm{Y}_{\mathrm{i}}(\tau, \phi)
$$

The average of the reaction rate for each species is shown as follows:

$$
\widetilde{\omega}=\int_{0}^{1} \int_{\tau}^{\infty} \tilde{P}(\tau, \phi) \omega_{\mathrm{Yi}_{1}}(\tau, \phi) \mathrm{d} \tau \mathrm{d} \phi
$$

The variables being independent, $\tilde{\mathrm{P}}(\tau, \phi)=\tilde{\mathrm{P}}(\tau) \tilde{\mathrm{P}}(\phi)$ are written what leads to:

$$
\overline{\rho \omega \mathrm{Y}_{\mathrm{i}}}=\bar{\rho} \int_{0}^{1} \tilde{\mathrm{P}}(\phi) \int_{\tau}^{\infty} \omega_{\mathrm{Y}_{1}} \tilde{\mathrm{P}}_{\tau}(\tau) \mathrm{d} \tau \mathrm{d} \varphi
$$

By considering a given particle since the idea of the model is to follow the history of it, the Eq. 2 lead to:

$$
\omega_{\mathrm{Y}}=\frac{1}{\tau}\left[\frac{\mathrm{dY}}{\mathrm{d} \phi}(\tilde{\phi}-\phi)-(\tilde{\mathrm{Y}}-\mathrm{Y})\right]
$$

The line portions corresponding to this rate of reaction are the mixing (segments $\mathrm{AD}$ and $\mathrm{BF}$ ), jump (segments $\mathrm{DE}$ and $\mathrm{FG}$ ) then equilibrium (segments $\mathrm{EC}$. and $\mathrm{GC}$ ). Mixing branch on the segments $\mathrm{AD}$ and $\mathrm{BF}$, particles do not react; the reaction rate zero:

$$
\omega_{\mathrm{Y}}^{\mathrm{MIX}}(\tau, \phi)=0
$$

Jump branch on the segments $\mathrm{DE}$ and $\mathrm{FG}$, the reaction rate is considered as follows:

$$
\omega_{\mathrm{Y}}^{\phi_{i g}^{+}}(\tau, \phi)=\tilde{\mathrm{P}}(\phi)\left(\frac{\tilde{\phi}-\phi_{\mathrm{ig}}^{+}}{\tau}\right) \delta \mathrm{Y}_{\phi_{\mathrm{ig}}^{+}}
$$

With,

$$
\begin{gathered}
\delta \mathrm{Y}_{\phi_{i g}^{+}}=\frac{\tilde{\mathrm{Y}}}{\tilde{\phi}} \phi_{\mathrm{ig}}^{+} \\
\omega_{\mathrm{Y}}^{\phi_{\mathrm{i}}}(\tau, \phi)=\tilde{\mathrm{P}}(\phi)\left(\frac{\tilde{\phi}-\phi_{\mathrm{ig}}^{-}}{\tau}\right) \delta \mathrm{Y}_{\phi \bar{g}}
\end{gathered}
$$

With,

$$
\begin{aligned}
& \delta \mathrm{Y}_{\phi_{\tilde{z}}}=\left(\frac{\mathrm{Y}_{\mathrm{MAX}}-\tilde{\mathrm{Y}}}{1-\tilde{\phi}}-\frac{\mathrm{Y}_{\mathrm{MAX}}}{1-\phi_{\mathrm{st}}}\right) \phi_{\mathrm{ig}}^{-}+ \\
& \left(1-\frac{\phi_{\mathrm{st}}}{1-\phi_{\mathrm{st}}}\right) \mathrm{Y}_{\mathrm{MAX}}-\frac{1}{1-\tilde{\phi}}\left(\mathrm{Y}_{\mathrm{MAX}}-\tilde{\phi}\right)
\end{aligned}
$$

Equilibrium branch on the segment GC, we have:

$$
\omega_{\mathrm{Y}}^{\mathrm{EQU}}(\tau, \phi)=-\frac{\left(\tilde{\mathrm{Y}}(1-\tilde{\phi})-\mathrm{Y}_{\mathrm{MAX}}\left(\tilde{\phi}-\phi_{\mathrm{st}}\right)\right)}{\tau\left(1-\phi_{\mathrm{st}}\right)}
$$

on segment $\mathrm{EC}$, since $\mathrm{Y}=0$ and $\mathrm{dy} / \mathrm{dZ}=0$, we have: 


$$
\omega_{\mathrm{Y}}^{\mathrm{EQU}}(\tau, \phi)=-\frac{\tilde{\mathrm{Y}}}{\tau}
$$

The instantaneous reaction rate is the sum of the three contributions as follows:

$$
\omega_{\mathrm{Y}}(\tau, \phi)=\underbrace{\omega_{\mathrm{Y}}^{\mathrm{MIX}}(\tau, \phi)}_{\text {mixing }}+\underbrace{\omega_{\mathrm{Y}}^{\phi_{\mathrm{ig}}^{+}}(\tau, \phi)+\omega_{\mathrm{i}}^{\phi_{\mathrm{i}}}(\tau, \phi)}_{\text {jump at } \phi_{\mathrm{ig}}^{+} \text {and } \phi_{\mathrm{ig}}^{-}}+\underbrace{\omega_{\mathrm{Y}}^{\mathrm{EQU}}(\tau, \phi)}_{\text {equilibrium }}
$$

The mean reaction rate is given by the following integral:

$$
\tilde{\omega}_{\mathrm{Y}}=\frac{\overline{\rho \omega \mathrm{Y}}}{\bar{\rho}}=\int_{0}^{1} \tilde{\mathrm{P}}(\phi) \int_{\tau}^{\infty} \omega_{\mathrm{Y}} \tilde{\mathrm{P}}_{\tau}(\tau) \mathrm{d} \tau \mathrm{d} \phi=\tilde{\omega}_{\mathrm{Y}}^{\mathrm{MIX}}+\tilde{\omega}_{\mathrm{Y}}^{\phi_{\mathrm{ig}}^{+}}+\tilde{\omega}_{\mathrm{Y}}^{\phi_{\mathrm{i}}^{\mathrm{i}}}+\tilde{\omega}_{\mathrm{Y}}^{\mathrm{EQU}}
$$

Expression of probability density function: The coupling between turbulence and chemistry results in the pdf appearing in the expression of the mean reaction rate, shown by the $\mathrm{MLL}$ model. $\mathrm{P}(\phi)$ who is the probability so that the mixing takes place is taken in this version of the model as being a function rectangle and peak give by Borghi and Moreau (1977); we know that this produced of the satisfactory results in its four configurations as had obtained Obounou et al. (1994). Let us specify that a more general formulation is in the use of the beta function Press et al. (1986). After studies undertaken by Fox (1995) then by Sabel'nikov and Gorokhovski (2001) on the introduction of a model with multiple scale considering the whole spectrum of the scales of time being located between the micro-scales and the integral scale it arises that $P_{\tau}\left(\tau^{*}\right)=\delta\left(\tau-\tau^{*}\right)$ with $\tau$ which is the scale of a mean mixing time so that the chemical reaction occurs and $\delta$ fine-grained pdf or probability density of a realization.

This use is justified by the fact that it avoids an integration moreover according to $\tau$. Being in a situation of lifted flame, in order to better represent the premixing which is done before effective combustion, we consider like a Log-normal function like Bray et al. (1984):

$$
\mathrm{P}_{\tau}=\frac{1}{\tau_{\mathrm{T}}-\tau_{\mathrm{K}}} \exp \left(\frac{\tau_{\mathrm{K}}-\tau}{\tau_{\mathrm{T}}-\tau_{\mathrm{K}}}\right)
$$

$$
\text { With } \quad \tau_{\mathrm{T}}=\frac{\mathrm{k}}{\varepsilon} \text { and } \tau_{\mathrm{K}}=\left(\frac{\vartheta}{\varepsilon}\right)^{\frac{1}{2}}
$$

Chemical kinetics: The determination of the ignition delays translating time necessary so that the ignition occurs, passes by the use of a kinetic mechanism. Knowing well that a global kinetic diagram with one equation shows only satisfactory results near to

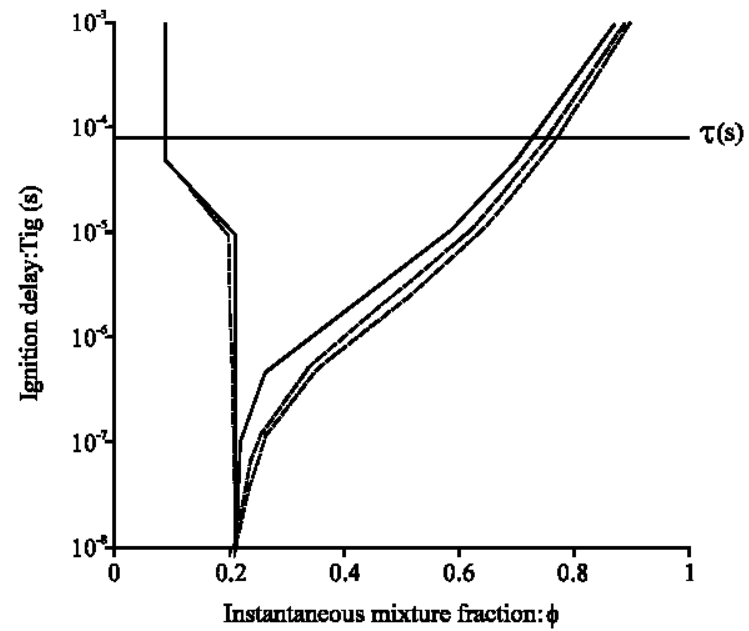

Fig. 3: Ignition delay versus instantaneous mixture fraction; $\tilde{\phi}=0.13, \mathrm{Y}_{\mathrm{O}_{z}}=0.002812 \quad$ (solid line), $\tilde{\mathrm{Y}}_{\mathrm{O}_{z}}=0.01095$ (dashed line), $\tilde{\mathrm{Y}}_{\mathrm{O}_{z}}=0.01637$ (dashdot line)

stoichiometry; Obounou et al. (1994) showed that more chemistry is detailed (complexes chemistry), more the precision of the chemical time is good in a rather broad range. Since here we are interested in the auto-ignition, the calculation retained for the tabulation of chemical times is of type PSR (Perfect Stirred Reactor), supporting a homogeneous autoignition; more the number of points taken for ranging between 0 and 1 is large, more the precision is good. The determination then the tabulation of these chemical delays are made by use of package Fortran CHEMKIN2 of the Sandia laboratory; the kinetic mechanism is described by the GRI 3.0 of Bowman et al. (1997) corresponding to the combustion of methane in the air which involves 5 elements, 53 species and 325 reactions. The delays are presented in the form of curves in U; Fig. 3 arises the profiles for pure oxidant then for pure fuel; ignition occur by comparison of this chemical time to the mixture time. Thus, it can predict partial extinctions when chemistry is slow (is located in top of the curves in U), we also captures finite chemistry (is located on the curves in $U$ ) then infinitely fast chemistry. On the basis of the variable of SchvabZeldovich, the mixture fraction for the configuration is shown by:

$$
\phi=\frac{2 \frac{\mathrm{M}_{\mathrm{O}_{\mathrm{z}}}}{\mathrm{M}_{\mathrm{CH}_{4}}} \mathrm{Y}_{\mathrm{CH}_{4}}-\mathrm{Y}_{\mathrm{O}_{\mathrm{z}}}-2 \frac{\mathrm{M}_{\mathrm{O}_{\mathrm{z}}}}{\mathrm{M}_{\mathrm{CH}_{4}}} \mathrm{Y}_{\mathrm{CH}_{4}}^{\text {collow }}+\mathrm{Y}_{\mathrm{O}_{\mathrm{z}}}^{\text {collow }}}{2 \frac{\mathrm{M}_{\mathrm{O}_{\mathrm{z}}}}{\mathrm{M}_{\mathrm{CH}_{4}}} \mathrm{Y}_{\mathrm{CH}_{4}}^{\text {fuel }}-\mathrm{Y}_{\mathrm{O}_{\mathrm{z}}}^{\text {fuel }}-2 \frac{\mathrm{M}_{\mathrm{O}_{\mathrm{z}}}}{\mathrm{M}_{\mathrm{CH}_{4}}} \mathrm{Y}_{\mathrm{CH}_{4}}^{\text {collow }}+\mathrm{Y}_{\mathrm{O}_{\mathrm{z}}}^{\text {collow }}}
$$

With stoichiometry, $\mathrm{Y}_{\mathrm{CH} 4}=\mathrm{Y}_{\mathrm{OZ}}=0$; what leads to $\phi_{\mathrm{st}}=0.1769$ similar that the value shown by the literature; the curves of the ignitions times are thus shifted towards this position. 


\section{RESULTS AND DISCUSSION}

Diagram of the computational domain: The field of calculation drawn then meshed by GAMBIT 2.4.6 is taken $2 \mathrm{D}$ axissymetric like Fig. 4; this field is then sub-divided in under fields in order to refining the grid in the interesting places such as in the layer of shearing. The researchers implemented the MIL model in the FLUENT 6.3.26 code (2006) through the UDF (User Define Functions) (2006) in order to carry out the numerical simulations.

Grid independence: Three grids were tested to determine the solution which does not depend on it any more; the details of the grid are shown in Table 2. Mesh 1 is lightest being used to gauge calculation; meshes 2 and 3 give close results and either may be used to produce a grid-independent solution. However, the finer mesh (Mesh 3) is selected here (although, mesh 2 is optimum) and is used in all subsequent calculations.

Results of numerical simulations: The turbulence model retained in FLUENT is the realizable K- $\varepsilon$. Researcher describes the results of simulation per use of the model

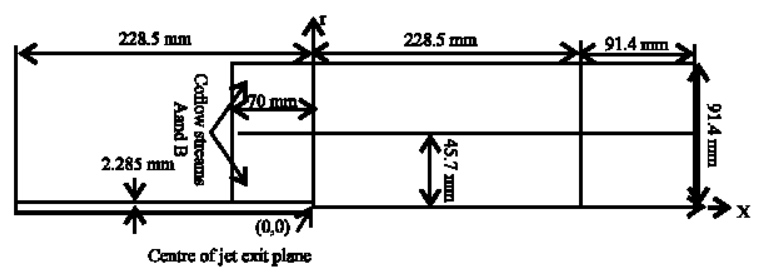

Fig. 4. Diagram of the computational domain

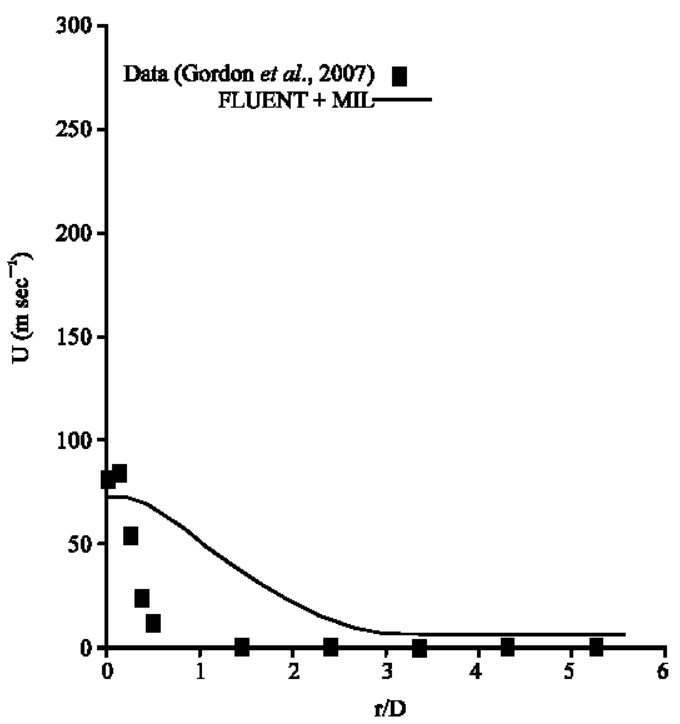

which researcher developed and implemented in FLUENT; a comparison is then made with experimental measurements. The assumption of 2D axissymetric simulation contrary to a case $3 \mathrm{D}$, product of the tiny differences since the parameters are realised. It is known that the RANS (Reynolds Average NavierStokes) simulation led to satisfactory results as in a recent calculation carried out by Mouangue and Obounou (2009). Figure 5 shows the evolution of the radial profile of mean velocity in $\mathrm{x} / \mathrm{D}=15$ and 30 . It arises that the $\mathrm{MLL}$ model lowers well the values which approach the experimental data; the bringing together is more effective as of the area of pre-flame with $\mathrm{x} / \mathrm{D}=30$. The MLL model by its aspects Intermittent and Lagrangian, makes it possible to better follow the comparison turbulencechemistry which translates the position of the flame better. This led on Fig. 6 and 7, respectively arising the

\begin{tabular}{lrrr}
\multicolumn{3}{l}{ Table 2: Details of the domain mesines } \\
\hline Parameters & Fuel jet & Coflow & Total cells \\
$\mathbf{x}$ & & & \\
From (mm) & -228.500 & -70.000 & - \\
To (mm) & 319.900 & 319.900 & - \\
$\mathbf{r}$ & & & \\
From (mm) & 0.000 & 2.285 & - \\
To (mm) & 2.285 & 91.400 & - \\
Mesh 1 & & & - \\
X & 55.000 & 45.000 & 1615 \\
Y & 13.000 & 20.000 & - \\
Mesh 2 & & & 6460 \\
X & 110.000 & 90.000 & \\
Y & 26.000 & 40.000 & - \\
Mesh 3 & & & 14,535 \\
X & 165.000 & 135.000 & \\
Y & 39.000 & 60.000 & \\
\hline
\end{tabular}

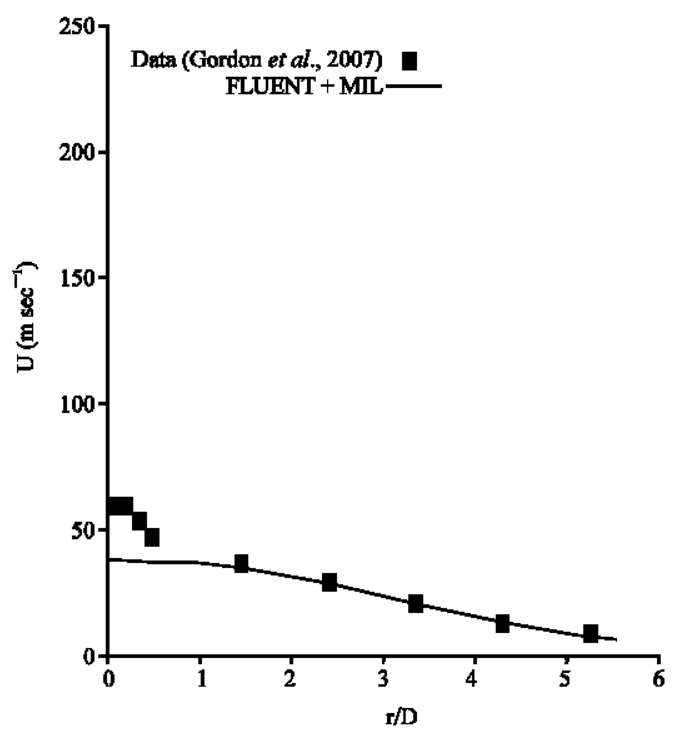

Fig. 5: Radial profiles of the mean velocity at 2 values of $\mathrm{x} / \mathrm{D}=15$ and 30 (from left to right); FLUENT+MIL (solid line), measurements (symbols) eddy dissipation (dashed line) 
position of the flame and the profiles of temperature. It is observed that the calculation is satisfactory; the radial profiles for temperature in Fig. 7 indicate no reaction

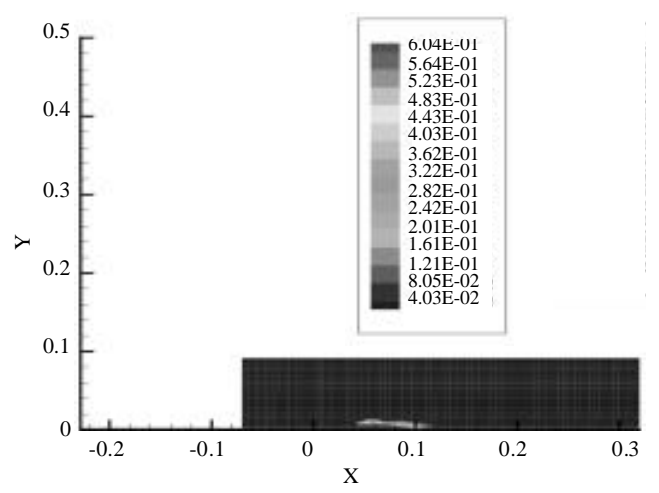

Fig. 6: Mean reaction rate field $\tilde{\omega}_{\mathrm{Y}}\left(\mathrm{s}^{-1}\right)$
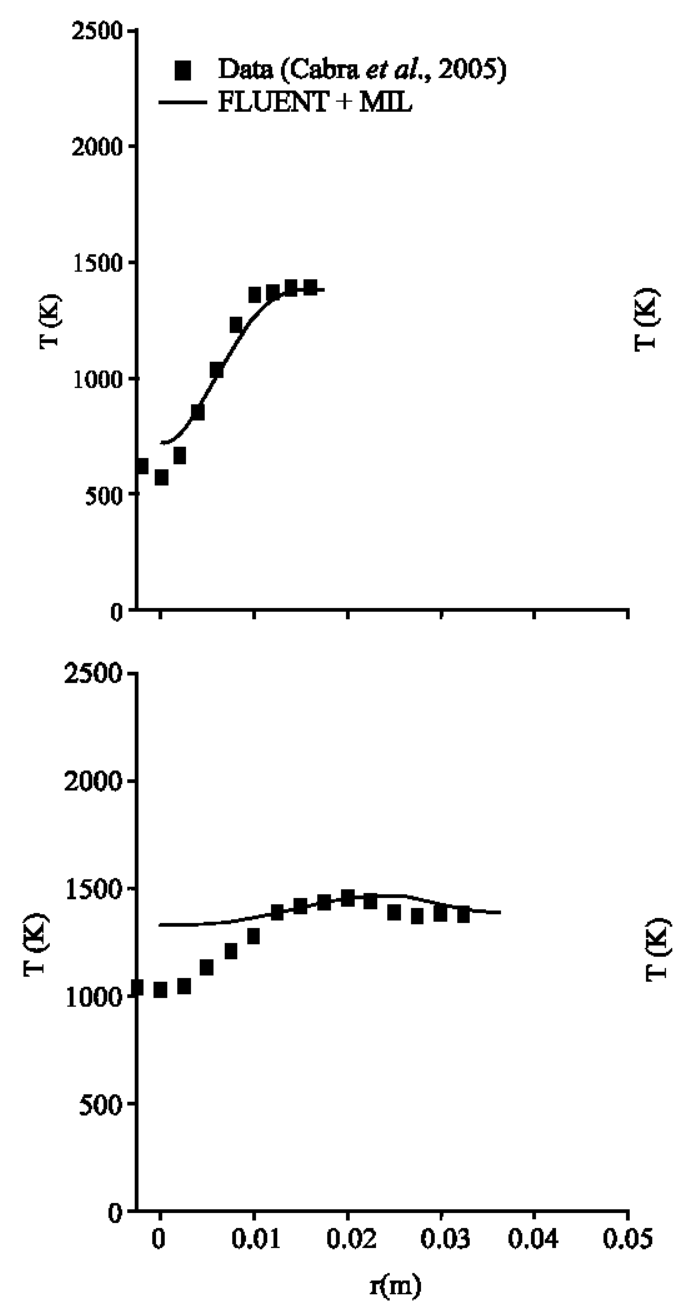

in the first 30 diameters from the exit nozzle. Initially, mixing without reaction occurs between the vitiated coflow and fuel jet. It is not until $\mathrm{x} / \mathrm{d}=40$ that we see a distinct increase in the temperature fluctuations and a rise in the mean temperature above the coflow condition.

The values remains close, one ignite when it is necessary and moreover, the liftoff height is well agree with experiments between $x / D=30$ and $x / D=40$.

In $x / D=50$, we observes a light shift resulting in the difference in positioning of bump materializing ignition; this comes owing to the fact that the model does not arise very well the premixing which takes place before effective combustion. The flame being lifted, the time of diffusion is more significant in the simulation compared to the experiment; the length of mixing is slightly larger in the calculation.
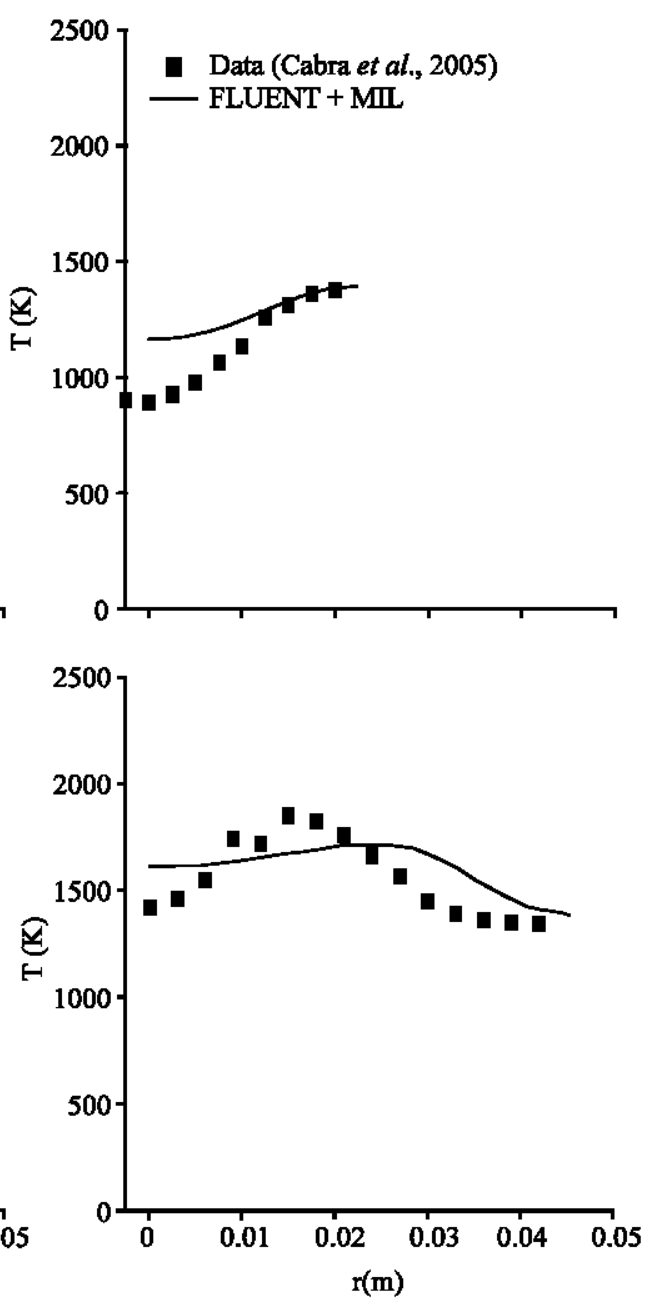

Fig. 7: Radial profiles of the mean temperature at 4 values of $x / D=15,30,40$ and 50 (from left to right then top to bottom); FLUENT+ML (solid line), measurements (symbols), eddy dissipation (dashed line) 


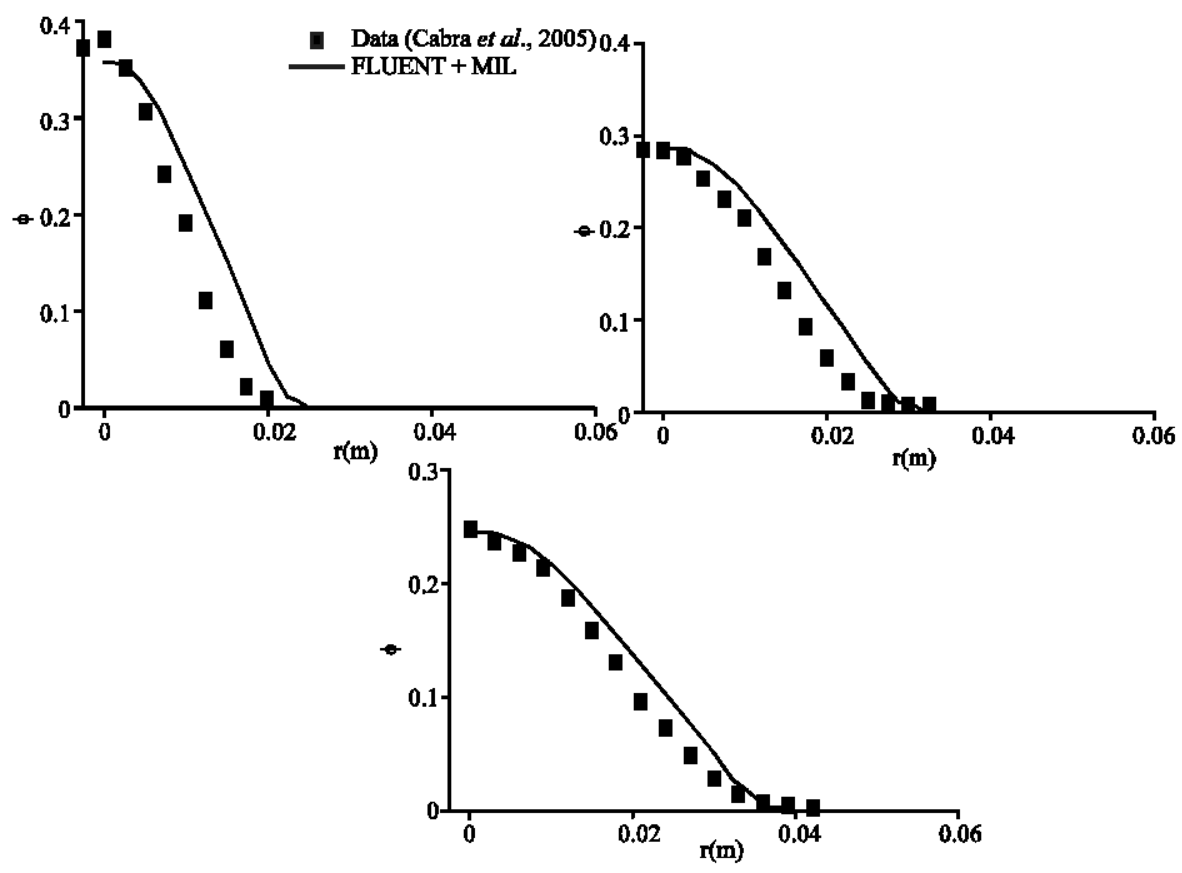

Fig. 8: Radial profile of the mean mixture fraction at 3 values of $x / D=30,40$ and 50 (from left to right then top to bottom); FLUENT + ML (solid line), measurements (synbols), eddy dissipation (dashed line)
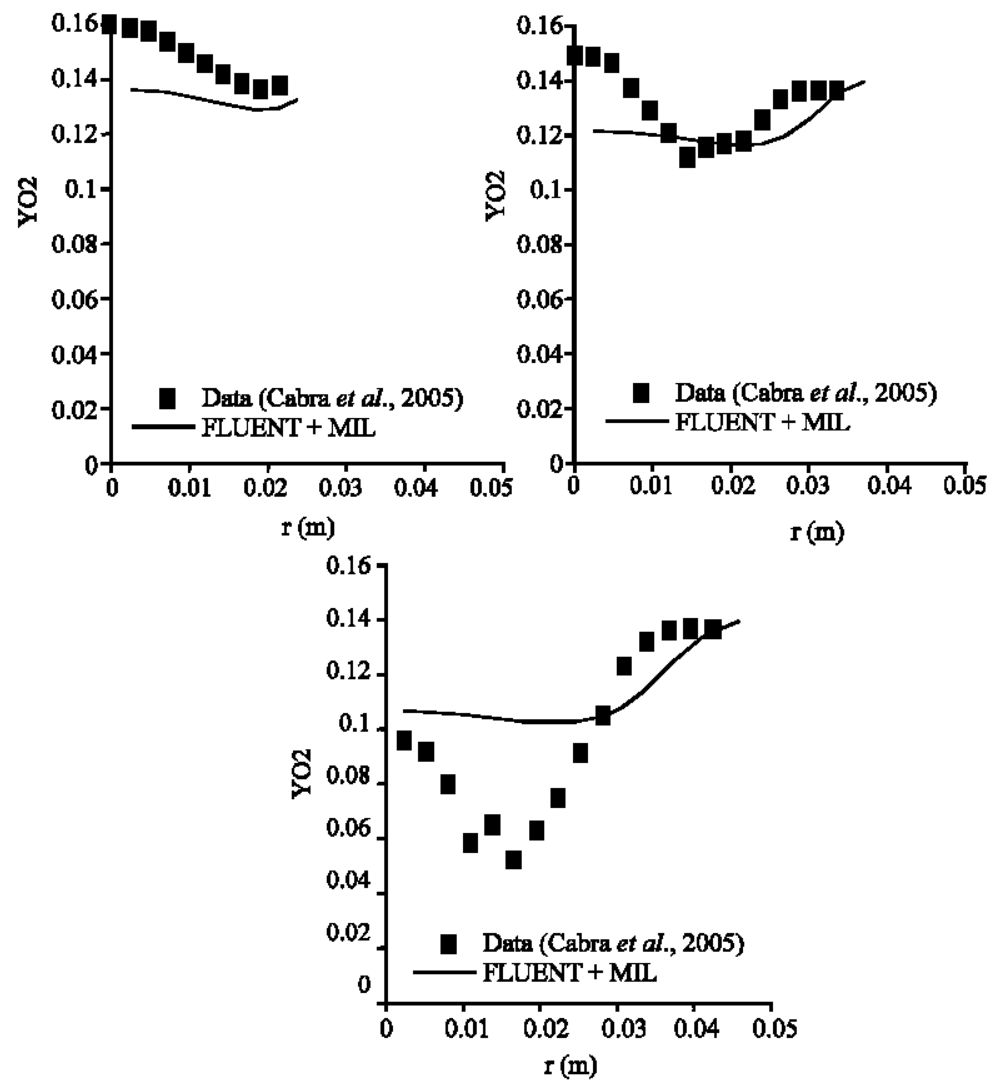

Fig. 9: Radial profile of the $\mathrm{o}_{2}$ mean mass fraction at 3 values of $\mathrm{x} / \mathrm{D}=30,40$ and 50 (from left to right then top to bottom); FLUENT + MLL (solid line), measurements (symbols), eddy dissipation (dashed line) 

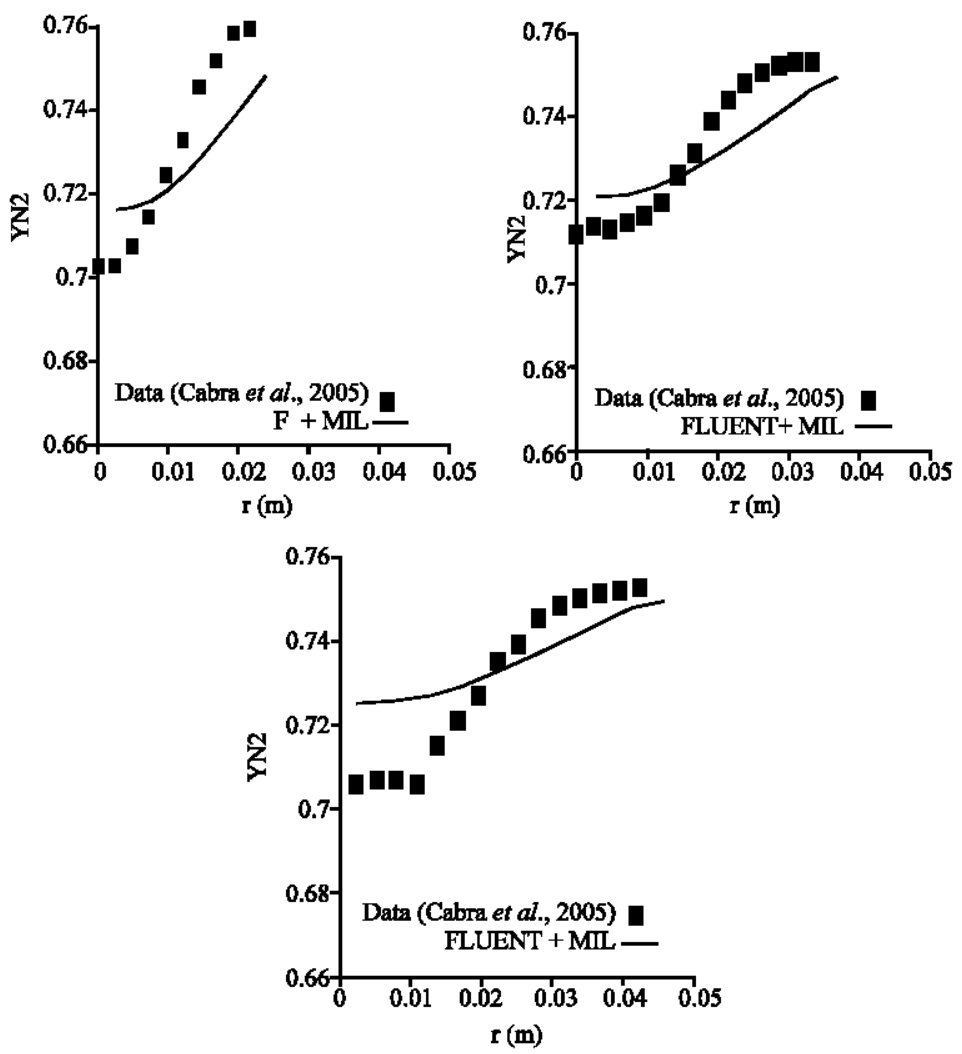

Fig. 10: Radial profiles of the $\mathrm{N}_{2}$ mean mass fraction at 3 values of $\mathrm{x} / \mathrm{D}=30,40$ and 50 (from left to right and top to bottm); FLUENT + MLL (solid line), measurments (symbols), eddy dissipation (dashed line)

Above the place of stabilization of the flame appears; this for a given kinetics. The flame is puffed up and we observes being separation towards a position enters $\$ \mathrm{x} / \mathrm{D}$ $=30 \$$ and $\$ \mathrm{x} / \mathrm{D}=40 \$($ Cabra et al., 2005) at the beginning of the calculation, the ignition of the flame occurs downstream of this steady state stabilization point. Afterward, during the convergence of the calculation, the flame front propagates toward this point. The reasons of this shift are confirmed on the curves of Fig. 8 shows the mixing fraction. The dynamics of the vortices strongly improves oxidant and fuel mixing; what is not taken into account in the calculation. We obtain nevertheless, the same evolutions. This influence is observed naturally on the profiles of the species, since it is they which mixing. Fig. 9 shows the radial profile of the average mass fraction of dioxygene. Because the model over-estimates the mixing layer, it predicts low values of the mass fraction with $x / D=30$ and 40 , then raised values with $x / D=50$. We have the same tendency on Fig. 10 which shows the evolution of the inert. In agreement with Cao et al. (2005), then Cabra et al. (2005), the turbulence intensity presents a bimodal form Fig. 11 shows this distribution. The bimodal distribution makes it possible the flame to be spread from the outside into the core area.

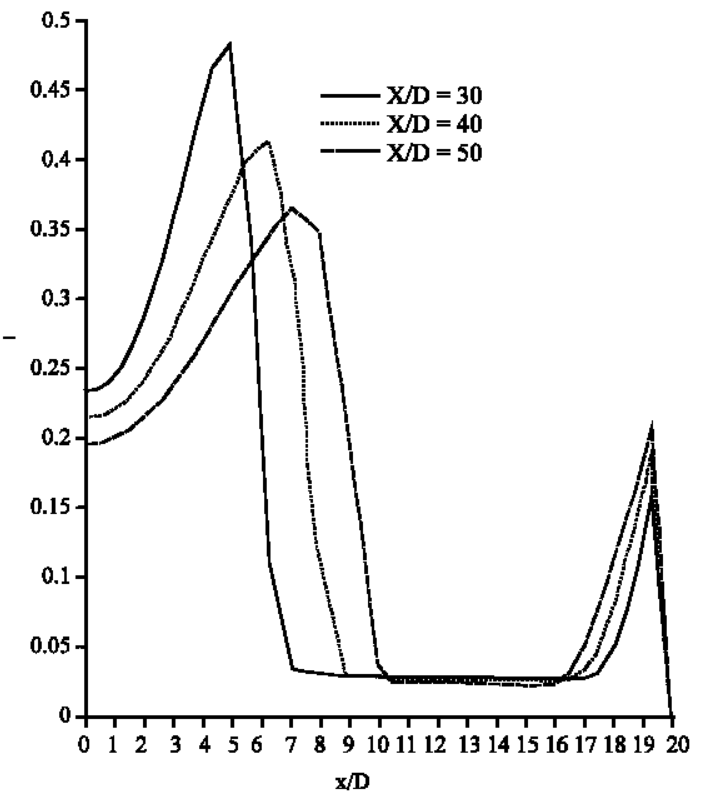

Fig. 11: Distribution of the turbulence intesity (FLUENT $+\mathrm{MLL}) ; \mathrm{x} / \mathrm{D}=30$ (solid line), $\mathrm{x} / \mathrm{D}=40$ (dashed line, $x / D=50$ (dashed line) 


\section{CONCLUSION}

The Lagrangian Intermittent Model was proposed with for objective describing auto-ignition then nonpremixed combustion and the partially premixed turbulent flames. Complex chemistry was tabulated based on a calculation PSR with mechanism GRI 3.0 for methane Bowman et al. (1997). Their implementation was made in the commercial code FLUENT with a formulation RANS; the simulation of the experiment of Cabra et al. (2005) of lifted flame to methane/air is produced. The comparison between calculation and experimental measurements in term of radial profile of mean velocity, temperature, from mixing fraction and average mass fraction of the species is arisen with an agreement; the liftoff is well captured. Concerning the perspective for improvement of this research in the short run, we will propose a calculation on this same flame using the version modified of the MLL model; this version, in addition to its capacity to take into account engines PSR or not already proved reliable within the framework of the lifted flames as in study of Mura and Demoulin (2007). The phenomenon of auto-ignition will be captured better by a calculation SENKIN which moreover, a study of sensitivity of the kinetic diagrams allows. It would be interesting to take a formulation of as a beta function which are more general than the rectangle and peak which were used. Moreover, the chemical we could be improved by taking tables of delays more discretized much using the more recent kinetic diagrams.

\section{ACKNOWLEDGEMENTS}

The researchers express their gratitude to Dr. Arnaud Mura from LCD UPR 9028 CNRS Poitiers for helpful discussions concerning the ML model, implementation via UDF of FLUENT and for their learning. Reseachers are also indebted to Ricardo Cabra and Prof. Robert Dibble (University of California) for providing experimental data.

\section{REFERENCES}

Borghi, R. and M. Gonzalez, 1986. Applications of Lagrangian models to turbulent combustion. Combust. Flame, 63: 239-250.

Borghi, R. and P. Moreau, 1977. Turbulent combustion in a premixed flow. Acta Astronaut, 4: 321-341.

Bowman, C.T., R.K. Hanson, W.C. Gardiner, V. Lissianski and M. Frenklach et al., 1997. An optimized detailed chemical reaction mechanism for methane combustion and no formation and reburning. Technical Report Gas Research Institute. Chicago IL. Report No. GRI-97/0020.

Bray, K.N.C., P.A. Libby and J.B. Moss, 1984. Scalar length scale variations in premixed turbulent flames. Symp. Int. Combust., 20: 421-427.
Cabra, R., T. Myhrvold, J.Y. Chen and R.W. Dibble, 2002. Simultaneous laser raman-rayleigh-lif measurements and numerical modeling result of a lifte turbulent $\mathrm{H}_{2} / \mathrm{N}_{2}$ jet flame in a vitialed coflow. Proc. Combust. Inst., 29: 1881-1888.

Cabra, R., J.Y. Chen, R.W. Dibble, A.N. Karpetis and R.S. Barlow, 2005. Lifted methane air jet flames in a vitiated coflow. Combust. Flame, 143: 491-506.

Cao, R.R., S.B. Pope and A.R. Masri, 2005. T urbulent lifted flames in a vitiated coflow investigated usind joint PDF calculations. Combust. Flame, 142: 438-453.

Domingo, P., L. Vervisch and D. Verynante, 2008. Largeeddy simulation of a lifted methane jet flame in a vitiated coflow. Combust. Flame, 152: 415-432.

Fox, R.O., 1995. The spectral relaxation model of the scalar dissipation rate in homogeneous turbulence. Phys. Fluids, 7: 1082-1094.

Gonzalez, L.F.M., R. Elamraoui and M. Obounou, 1997. Modelling finite-rate chemistry effects in nonpremixed turbulent combustion : Tet on the bluffbody stabilized flame. Combust. Flame, 110: 298-314.

Gonzalez, M. and R. Borghi, 1991. A Lagrangian Intermittent Model for Turbulent Combustion Theorical Basis and Comparisons with Experiments. Springer-Verlag, Berlin, pp: 293.

Gordon, R.L., A.R. Masri, S.B. Pope and G.M. Goldin, 2007. A numerical study of auto-ignition in turbulent lifted flames issuing into a vitiated co-flow. Combust. Theor. Modell., 11: 351-376.

Gordon, R.L., A.R. Masri and E. Mastorakos, 2008. Simultaneous Rayleigh temperature, $\mathrm{OH}$-and $\mathrm{CH}_{2} \mathrm{O}-$ LIF imaging of methane jets in a vitiated coflow. Combust. Flame, 155: 181-195.

Masri, A.R., R.R. Cao, S.B. Pope and G.M. Goldin, 2003. Pdf calculations of turbulent lifted flames of $\mathrm{H}_{2} / \mathrm{N}_{2}$ fuel issuing into a vitiated co-flow. Combust. Theor. Modell., 8: 1-22.

Michel, J.B., O. Colin, C. Angelberger and D. Veynante, 2009. Using the Tabulated diffusion flamelet model $\mathrm{ADF}-\mathrm{PCM}$ to simulate a lifted methane air jet flame. Combust. Flame, 156: 1318-1331.

Mouangue, R. and M. Obounou, 2009. Numerical simulation of turbulent diffusion flames of $\mathrm{H}_{2} /$ air. Phys. Chem. News, 50: 69-78.

Mura, A. and F.X. Demoulin, 2007. Lagrangian intermittent modelling of turbulent lifted flames. Combust. Theor. Modell., 11: 227-257.

Obounou, M., M. Gonzalez and R. Borghi, 1994. A lagrangian model for predicting turburlent diffusion flames with chemical kenetic effects. Proc. Combust. Inst., 25: 1107-1113.

Patwardhan, S.S., S. De, K.N. Lakshmisha and B.N. Raghunandan, 2009. CMC simulations of lifted turbulent jet flame in a vitiated cofow. Proc. Combust. Inst., 32: 1705-1712. 
Press, W.H., B.P. Flannery, S.A. Teukolsky and W.T. Vetterling, 1986. Numerical Recipes in Fortran: The Art of Scientific Computing. Cambridge University Press, Cambridge.

Sabel'nikov, V. and M. Gorokhovski, 2001. The new development of the LMSE mixing model and its application to the turbulent mixing of scalar in the presence of evaporating droplets. Proc. Turbulent Shear Flow Phenomena Conf. TSFP2, 3: $257-262$.
Villermaux, J. and J.C. Devillon, 1972. Representation de la coalescence et de la redispersion des domaines de segregation dans un fluide par un modele d'interaction phénoménologique. Proceedings of the International Symposium on Chemical Reaction Engineering, May 2-4, Elsevier, Amsterdam, The Netherland, pp: 1-13.

Wang, Z.H., J.R. Fan, J.H. Zhou and K.F. Cen, 2007. Direct numerical simulation of hydrogen turbulent lifted jet flame in a vitiated coflow. Chinese Sci. Bull., 52: $2147-2156$. 